\title{
Belphégor
}

\section{Disparitions et réapparitions, mort et renaissance : les traductions fantasques de Marcel Duhamel}

\author{
Alistair Rolls, Clara Sitbon and Marie-Laure Vuaille-Barcan
}

\section{(2) OpenEdition}

\section{Journals}

\section{Electronic version}

URL: http://journals.openedition.org/belphegor/735

DOI: 10.4000/belphegor.735

ISSN: 1499-7185

Publisher

LPCM

\section{Electronic reference}

Alistair Rolls, Clara Sitbon and Marie-Laure Vuaille-Barcan, « Disparitions et réapparitions, mort et renaissance : les traductions fantasques de Marcel Duhamel », Be/phégor [Online], 14 | 2016, Online since 10 October 2016, connection on 02 May 2019. URL : http://journals.openedition.org/ belphegor/735; DOI : 10.4000/belphegor.735

This text was automatically generated on 2 May 2019.

\section{$\Theta \Theta \Theta$}

Belphégor est mis à disposition selon les termes de la Licence Creative Commons Attribution - Pas d'Utilisation Commerciale - Pas de Modification 4.0 International. 


\title{
Disparitions et réapparitions, mort et renaissance : les traductions fantasques de Marcel Duhamel
}

\author{
Alistair Rolls, Clara Sitbon and Marie-Laure Vuaille-Barcan
}

L'année 2000 vit la publication en France du roman 1280 âmes. Malgré les apparences, ce nouveau texte n'était pas la traduction littérale du classique Pop 1280 de Jim Thompson. Ce roman de Jean-Bernard Pouy se donnait pour mission de résoudre l'énigme de la disparition de cinq personnes dans le titre de la célèbre traduction de Marcel Duhamel, intitulée 1275 âmes et devenue pour ainsi dire un classique français à part entière. Le héros de Pouy, un libraire parisien nommé de manière facétieuse Pierre de Gondol, est un érudit qui aime répondre aux questions les plus diverses de ses clients. Un jour, l'un d'eux lui demande de retrouver ces cinq âmes manquantes. Comme si ses connaissances encyclopédiques en littérature n'étaient pas suffisantes pour trouver la solution en restant en France, de Gondol décide d'entreprendre un voyage en voiture en Amérique en prenant le prétexte de retrouver sa petite amie actrice qui est sur le point de faire une tournée outre-Atlantique. Il semble qu'il veuille retracer le parcours du texte, le lire à rebours, dans le sens opposé à celui de la traduction de l'américain vers le français. De Gondol choisit d'ignorer la cause la plus probable au changement de titre en français, qui serait de dire que la traduction, en tant qu'acte postérieur à l'achèvement du texte original, choisit de montrer son statut de texte traduit en calculant la population après que les meurtres originaux ont été commis dans le roman de Thompson. Il considère que le nombre de pertes dans le titre de Duhamel - cinq âmes - est trop bas ou, au contraire, trop élevé : les victimes noires sont-elles incluses dans le compte, alors que leur statut d'êtres humains avec des âmes est contesté par les bons citoyens (blancs) de Pottsville dans le texte original ${ }^{1}$ ? La solution, découverte quand le libraire reconstitue l'original du roman lors de son road story aux États-Unis, résiderait dans le fait que certains passages ont été supprimés dans la première traduction. Le roman de Pouy est donc apparemment fondé sur les pratiques traductives de Marcel Duhamel, dont le célèbre 1275 âmes a paru dans la Série Noire en 1966, trois ans après la publication de Pop 1280 de Thompson. Il semble donc que Pouy souligne dans sa propre réécriture en français ce que beaucoup 
d'autres, comme Marc Lapprand'2, avaient déjà remarqué, c'est-à-dire que les traductions de la Série Noire et peut-être particulièrement celles de Duhamel lui-même, étaient des adaptations très libres, en raison du manque de considération accordée au genre, des restrictions en termes de nombre de pages ou de règles plus ou moins tacites sur les coupes acceptables pour donner la primauté à l'action ${ }^{3}$.

2 Cependant, si l'on compare plusieurs éditions, on se rend compte que le texte auquel Pierre de Gondol se réfère pour analyser les coupes effectuées par Duhamel n'est pas celui du célèbre numéro 1000 de la Série Noire de 1966; en effet, malgré les connaissances approfondies de l'histoire de la Série Noire qu'il possède, le protagoniste de Pouy fonde son hypothèse sur une lecture du numéro 337 du Carré Noir, texte qui date de 1988. Or, vu l'expérience des pratiques du monde de l'édition français qu'a manifestement JeanBernard Pouy, dont le protagoniste n'est sans doute qu'un double fictionnel, il nous semble improbable qu'il ne soit pas conscient des différences qui peuvent exister entre les diverses rééditions des titres de la Série Noire, qu'ils renaissent dans la même série ou dans d'autres. Si Pierre choisit donc de consulter le texte du Carré Noir plutôt que celui emblématique - de la Série Noire, il se pourrait que ce soit pour nous induire en erreur en toute connaissance de cause, ou encore pour nous mettre la puce à l'oreille... Il nous semble difficile de croire qu'il aurait pris le premier livre qu'il avait sous la main; il s'agirait d'un acte opportun, beaucoup trop opportun, ou comme il le dit lui-même, en parodiant Cyrano, à propos de la traduction du Carré Noir : «Court et efficace. Un peu court, jeune homme $»^{4}$.

Or, une lecture de la traduction de Duhamel parue en Série Noire révèle effectivement que le passage (celui auquel Pierre s'intéresse et dans lequel figurent deux des âmes absentes, plus un cheval...), qui manque en 1988 manquait aussi en 1966, ce qui donne raison au libraire de Pouy. Mais le mystère ne s'arrête pas là. En consultant la traduction de Marcel Duhamel de 1275 âmes, pris au hasard sur les étagères d'une librairie à Paris, on n'est jamais sûr de tomber sur le même texte, et ce pour une raison des plus curieuses : le roman a été réédité en Série Noire en 1995 lors du cinquantenaire de la série et, fait rarissime, s'est vu accorder un nouveau numéro, le 2391, tout en gardant le même titre, à savoir 1275 âmes $^{5}$. Si bien qu'au moment de la parution de 1280 âmes de Pouy, il existe bel et bien deux versions de 1275 âmes en Série Noire. Dans l'une d'entre elles, les personnages repérés par Pierre de Gondol dans l'original de Thompson ont effectivement disparu, tandis que dans l'autre ils sont bien présents. S'agit-il d'une retraduction partielle, d'une coupe colmatée a posteriori suite à la découverte d'un chercheur, à l'instar des investigations mises en scène dans 1280 âmes ? Et quant au passage absent de l'édition de 1966 mais qui est ajouté à celle de 1995, s'agit-il d'une traduction faite (et puis omise) par Duhamel, par le chercheur mentionné par Pouy, ou encore par une tierce personne ? On sait que cette tendance qu'avaient Duhamel et ses collaborateurs à prendre certaines libertés avec le texte d'origine a été à l'origine d'une vague de retraductions : deux autres romans de Thompson qui avaient été tronqués en Série Noire ont été retraduits récemment et publiés chez Rivages : The Killer inside me (L'assassin qui est en moi) par JeanPaul Gratias, et The Getaway (L'Echappée) par Pierre Bondil. Tout ce que l'on peut dire avec certitude à propos de 1275 âmes est que la décision a été prise de réhabiliter le passage d'abord omis, de réinsérer les âmes qui manquaient, mais de garder le titre français choisi par Marcel Duhamel. Qu'il y ait 1275 ou 1280 âmes à Pottsville, une fois que leur histoire est traduite en français, elle endosse une toute autre personnalité. Nonobstant les découvertes de Pierre de Gondol, qui s'avèrent à la fois concluantes et partielles, nous 
aimerions avancer une explication plus simple du choix du titre de 1275 âmes. Et s'il indiquait tout bonnement "l'originalité » du texte français et la nouvelle vie dans son nouveau contexte ainsi accordée à l'œuvre originale grâce au geste traductif ? ${ }^{6}$

Comme nous l'avons noté ailleurs, les libraires font souvent d'excellents protagonistes parodiques dans le roman noir?. Lee Anderson de Vernon Sullivan dans J'Irai cracher sur vos tombes, par exemple, effectue lui aussi un voyage de Paris jusque dans le sud des ÉtatsUnis. Une lecture attentive permet de voir que le pastiche que fait Sullivan de la Série Noire - fondé sur la célèbre collection, comme celui de Pouy, mais publié par une autre maison d'édition - traite beaucoup moins du phénomène du roman noir né aux Etats-Unis que du milieu de l'édition française des années de l'immédiat après-guerre, phénomène que l'expression «traduit de l'américain» résume de manière à la fois parfaite et trompeuse 8 .

5 Il est bien connu que Duhamel a lancé la Série Noire en 1945 avec sa traduction de ce classique du genre Poison Ivy de Peter Cheyney, ou, en français, La Môme vert-de-gris. Ce que l'on sait bien également - en France du moins, où le nom de Cheyney est toujours célèbre, ce qui est moins le cas en Angleterre ou aux Etats-Unis, malgré l'intérêt récent que lui portent des spécialistes de la littérature policière comme Stephen Knight ${ }^{9}$ - est que Cheyney était anglais, et non américain (ainsi que le montre la couverture de La Môme vert-de-gris, le texte est depuis le début signalé comme étant traduit de l'anglais). Et pourtant, le mythe d'un genre forgé en traduction subsiste jusqu'à ce jour, avec l'importation en France de classiques américains conçus pour être lus par des lecteurs français souhaitant s'immerger dans des histoires criminelles situées aux Etats-Unis. La véritable révélation de La Môme vert-de-gris est que cette traversée transatlantique, comme le road story à l'envers de Pouy, n'a en fait pas lieu du tout dans les textes pionniers de la Série Noire. Le coup magistral de Duhamel avec La Môme vert-de-gris est, paradoxalement, de faire un minimum d'adaptation du roman de Cheyney et de laisser le texte original parler en français à son nouveau public de son propre contexte (français). Et c'est précisément avec ces mots introducteurs - La Môme vert-de-gris - que Duhamel adapte, plutôt qu'il ne traduit, cachant ainsi, au moment où il la découvre, la vraie signification de la môme Carlotta. En effet, Duhamel, en choisissant les trois mots initiateurs de la Série Noire, et donc en refusant de traduire littéralement le titre de Cheyney, Poison Ivy ${ }^{10}$, surnom de Carlotta, replace l'enquête du détective privé Lemmy Caution en France à la transition entre l'Occupation et la Libération: la môme est, bien sûr, un synonyme de la gosse (le terme préféré de Vernon Sullivan, bien que ce soit un gosse dans son cas), ou plus typiquement, de la souris ; le vert-de-gris est, pour sa part, la couleur spécifique des uniformes portés par les forces d'Occupation allemandes à Paris pendant les années noires. Et les souris grises étaient les auxiliaires féminines de l'armée allemande, surnommées ainsi parce qu'habillées de tailleurs gris. Dans la traduction de Duhamel, la souris aux yeux verts de Cheyney, devient l'héroïne éponyme à défaut d'être le personnage principal dans l'histoire et prend ainsi beaucoup plus d'importance par ce changement de titre. A la fin du livre, elle perdra ses attributs gris et le détective Lemmy Caution aura même l'impression de voir ses yeux changer de couleur, quand elle se montrera sous son vrai jour : elle demandera au gangster Rudy de lui lancer son revolver pour en finir elle-même avec le détective mais en fait mettra le gangster en joue.

6 En effet, Carlotta s'avère être un agent double, membre de la Résistance et ainsi jouant un rôle essentiel en tant que Française dans la victoire des Alliés. Elle est, finalement, une môme, ou une souris, et le gris n'est qu'une couverture. L'identité américaine se révèle 
française, et c'est le message de Duhamel : à la Libération, quand les blessures sont toujours trop à vif pour être apaisées, le passé trop récent pour être rapporté littéralement, on doit trouver un autre moyen pour l'exprimer. Et ce moyen, d'identité française, c'est ce qu'offrent les premières « traductions » de la Série Noire. Loin de n'être que des histoires américaines qui seraient reçues et lues de manière passive par des Français fraîchement libérés par les troupes alliées, ce sont des allégories d'une nouvelle identité nationale française traduites de façon active, dans un acte de résistance littéraire, dans le cadre d'une nouvelle série, noire et française. Ainsi, l'expression «traduit de l'américain » a été apposée a posteriori aux trois premiers textes, devenus cultes, de la Série Noire (La Môme vert-de-gris et Cet homme est dangereux de Peter Cheyney en 1945, et Pas d'orchidées pour Miss Blandish de James Hadley Chase en 1946), sur des textes qui étaient en réalité anglais, et ces titres pionniers de la Série Noire étaient, malgré les apparences, tout ce qu'il y a de plus français ${ }^{11}$.

\section{NOTES}

1. Jean-Bernard Pouy, 1280 âmes (Paris : Éditions Baleine / Le Seuil, 2000), p. 46-47.

2. Voir Marc Lapprand, «Les Traductions parodiques de Boris Vian », The French Review, 65, 4 (1992), 537-46.

3. Voir aussi l'article « Polars américains : la traduction était trop courte » de Jérôme Dupuis, du 24/10/2012 (http://www.lexpress.fr/culture/livre/polars-americains-la-traduction-etait-tropcourte_1178207.html).

4. 1280 âmes, p. 67.

5. Par contre, Pas d'orchidées pour Miss Blandish de James Hadley Chase, qui a été traduit par Duhamel et publié en 1946, devenant ainsi le numéro 3 de la Série Noire, a été réédité en 1962 dans la même série, dans une nouvelle traduction de Noël Chassériau et avec un nouveau numéro, le 719, changement qui a nécessité un nouveau titre : la nouvelle édition s'intitule Pas d'orchidées tout court.

6. Nous voudrions remercier Samuel Schwiegelhofer de la Bilipo et Matthieu Letourneux de l'Université Paris Ouest, pour leur aide précieuse, ainsi que le libraire Olivier Ancel de l'Amour du noir qui nous a encouragés à lire - et qui nous a vendu - 1280 âmes.

7. Voir, par exemple, Marie-Laure Vuaille-Barcan, Clara Sitbon and Alistair Rolls, " Jeux textuels et paratextuels dans J'irai cracher sur vos tombes: au-delà du canular ", Romance Studies, 32, 1 (2014), 16-26.

8. Ainsi le texte de Vernon Sullivan, ou plutôt de Boris Vian, exploite-t-il, de façon autoréflexive, ce renversement de polarité, et pourtant Pierre Bayard considère qu'il s'agit bien d'un texte de Sullivan l'Américain. Voir l'étude de Bayard dans Et si les œuvres changeaient d'auteur (Paris : Les Editions de Minuit, 2010).

9. Voir, par exemple, l'introduction de Secrets of Crime Fiction Classics: Detecting the Delights of 21 Enduring Stories (Jefferson, NC: McFarland \& Company, 2015), p. 7.

10. Littéralement le sumac vénéneux, plante sauvage d'Amérique du Nord. Un autre titre possible aurait pu être « Une plante vénéneuse ».

11. Cheyney et Chase ont largement dominé les premières années de la Série Noire. L'action de Cet homme est dangereux se passe notamment en Angleterre. Miss Blandish offre une allégorie de 
la France séquestrée qui renaît à travers sa fille dans La chair de l'orchidée en 1948. Voir Alistair Rolls et Deborah Walker, French and American Noir: Dark Crossings (Houndmills: Palgrave Macmillan, 2009), pp. 57-78. 